\title{
Crystal structure of Lamellipodin implicates diverse functions in actin polymerization and Ras signaling
}

\author{
Yu-Chung Chang, Hao Zhang, Mark L. Brennan, Jinhua Wu ${ }^{\bowtie}$ \\ Department of Developmental Therapeutics, Fox Chase Cancer Center, 333 Cottman Avenue, Philadelphia, PA 19111, USA \\ $\triangle$ Correspondence: Jinhua.wu@fccc.edu
}

Received August 25, 2012 Accepted September 20, 2012

\begin{abstract}
The adapter protein Lamellipodin (Lpd) plays an important role in cell migration. In particular, Lpd mediates lamellipodia formation by regulating actin dynamics via interacting with Ena/VASP proteins. Its RA-PH tandem domain configuration suggests that like its paralog RIAM, Lpd may also mediate particular Ras GTPase signaling. We determined the crystal structures of the Lpd RA-PH domains alone and with an $\mathrm{N}$-terminal coiled-coil region (cc-RA-PH). These structures reveal that apart from the anticipated coiled-coil interaction, Lpd may also oligomerize through a second intermolecular contact site. We then validated both oligomerization interfaces in solution by mutagenesis. A fluorescence-polarization study demonstrated that Lpd binds phosphoinositol with low affinity. Based on our crystallographic and biochemical data, we propose that Lpd and RIAM serve diverse functions: Lpd plays a predominant role in regulating actin polymerization, and its function in mediating Ras GTPase signaling is largely suppressed compared to RIAM.
\end{abstract}

KEYWORDS Lamellipodin, crystal structure, RIAM, coiledcoil, oligomerization

\section{INTRODUCTION}

Cell migration and adhesion are actin-dependent processes essential for cell differentiation, tissue formation, organism development, and morphogenesis. Enhancement of cell migration and reduction of cell adhesion are the key characteristics of malignant cell metastasis. Cell migration is governed by actin polymerization or exocytosis-induced cell extension at the leading edge. Cell adhesion is driven by the binding of a group of actin skeleton-associating proteins, called cell adhesion molecules, to the extracellular matrix. Lamellipodin (Lpd), an MRL (Mig10/RIAM/Lpd) family protein, has been shown to promote lamellipodia protrusion in fibroblast by interacting with Ena/NASP proteins (Krause et al., 2004). Its homologous protein RIAM (Rap1 Interacting Adapter Molecule) is also capable of regulating cell migration and has been shown to mediate Rap1-induced cell adhesion (Lafuente et al., 2004; Lee et al., 2009). Thus, these adapter proteins appear to be the linkage between upstream Ras signaling and actin dynamics. Apart from regulating actin dynamics, Lpd also exhibits other unique enzymatic functions including its catalytic activity of Butyrylcholinesterase, a potent therapeutic treatment targeting cocaine abuse (Li et al., 2008).

Lpd and other MRL proteins share a common structural architecture, including a central structural unit consisting of a Ras-associating (RA) domain and a pleckstrin homology $(\mathrm{PH})$ domain, an upstream coiled-coil region, and a number of polyproline motifs (Fig. 1A) (Colo et al., 2012). Furthermore, the two mammalian orthologs, Lpd and RIAM, both contain a helical region at the amino terminus for talin binding (Lee et al., 2009). RA and PH form a tandem domain pair (RA-PH), and serve tightly coordinated functions in both Ras GTPase signaling via the RA domain and membrane translocalization via the $\mathrm{PH}$ domain (Depetris et al., 2009; Wynne et al., in press). In the case of RIAM, structural and mechanistic studies revealed that the $\mathrm{RA}$ and $\mathrm{PH}$ domains function as a proximity detector for Rap1 GTPase at the plasma membrane, leading to insideout integrin activation (Wynne et al., in press). The proline-rich region recruits actin-binding proteins such as Ena/VASP and profilin (Krause et al., 2004; Lafuente et al., 2004). Ena/VASP proteins tetramerize through a coiled-coil region, thus facilitating actin binding and bundling (Krause et al., 2003). In mouse melanoma cells, knockdown of Lpd impairs lamellipodia forma- 
A

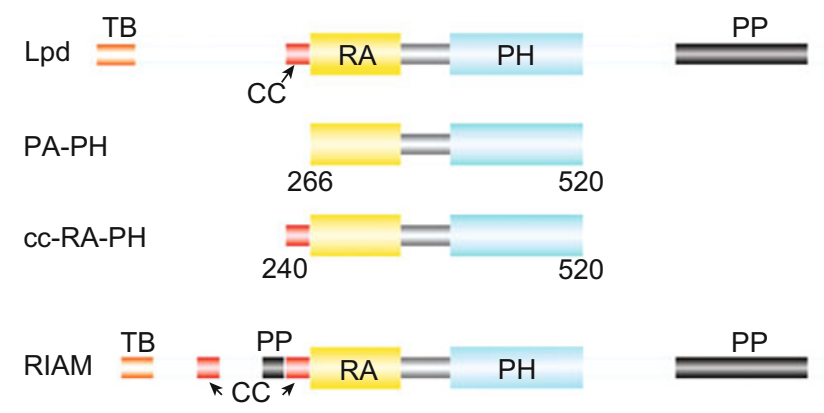

B

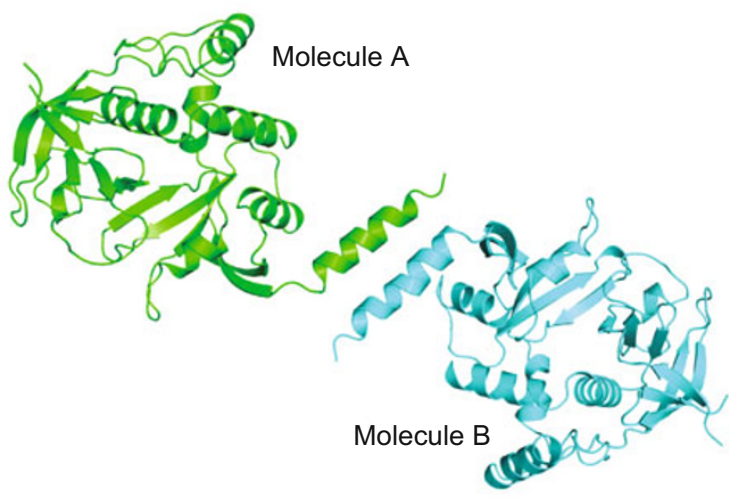

C

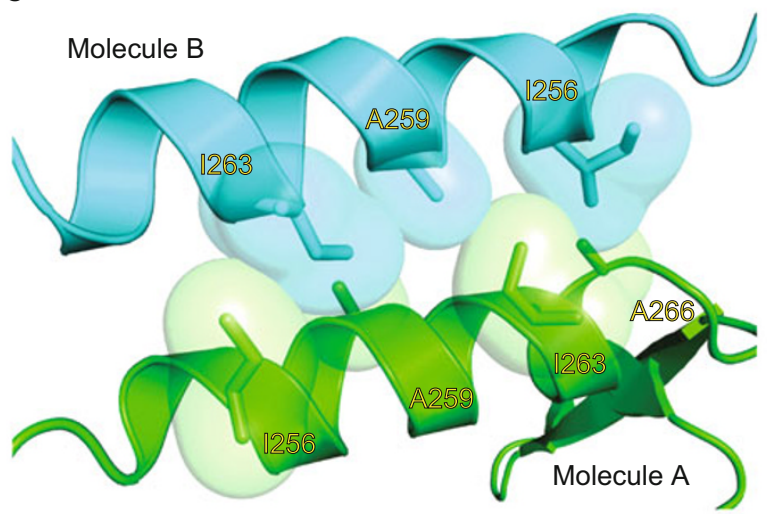

Figure 1. Structure of Lpd cc-RA-PH revealed a coiled-coil contact interface. (A) Schematic representation of Lpd and RIAM. RA, Ras-association domain; $\mathrm{PH}$, pleckstrin homology domain; PP, poly-proline region; CC, coiled-coil region; TB, talin-binding region. Two Lpd constructs are shown with their boundaries. (B) Ribbon diagram representation of Lpd cc-RA-PH non-crystallographic dimer. Two molecules interacting through an anti-parallel coiled-coil are colored in green and cyan. (C) Surface and stick representations of the hydrophobic interface at the coiled-coil contact site mediated by alanine and isoleucine residues.

tion, which is not seen in Ena/VASP knockout cells, suggesting Lpd is essential for regulating the actin cytoskeleton (Krause et al., 2004). Although the role of the coiled-coil region (cc) in Lpd has yet to be determined, the apparent oligomerization function indicates it may function in clustering Ena/VASP proteins, thereby enhancing actin polymerization and inducing lamellipodia and filopodia formation.

Here we present the crystal structures of the Lpd RA-PH tandem domain pair and an Lpd construct cc-RA-PH that contains the coiled-coil region and the RA-PH domain pair. The crystal structure of Lpd cc-RA-PH revealed a conventional intermolecular interaction mediated by the coiled-coil region. Surprisingly, there is a second, unique intermolecular contact site found in both Lpd cc-RA-PH and RA-PH structures. In vitro mutation analyses confirmed that both contact sites contribute to Lpd oligomerization, and the second site requires the integrity of the coiled-coil region. Oligomerization was not observed in a RIAM construct equivalent to Lpd cc-RA-PH, suggesting a lack of (or a significantly reduced) function in Ena/VASP clustering of RIAM. We also measured the phosphoinositide affinity to the Lpd PH domain. The weak binding affinity for phosphoinositides (compared to RIAM) indicates a functional deficiency of Lpd in Rap1 signaling and integrin-induced cell adhesion.
Overall, our data provide structural and biochemical basis that leads to the hypothesis that Lpd and RIAM serve diverse functions in facilitating Ena/VAP clustering and integrin activation, respectively, despite their similar structural architecture.

\section{RESULTS}

\section{Crystal structures of Lpd cc-RA-PH and RA-PH}

Lpd contains a talin-binding site near the amino terminus (Lee et al., 2009), a putative coiled-coiled region upstream of the central RA and $\mathrm{PH}$ domains, and a number of poly-proline regions that are responsible for interacting with actin-binding proteins, such as Ena/VASP and profilin (Krause et al., 2004). To establish the structural basis of Lpd in actin dynamics and Ras signaling, we engineered two Lpd constructs to encode residues 266-520 (RA-PH) of the Lpd RA-PH domain pair and residues 240-520 (cc-RA-PH) comprising the coiled-coil region and the RA-PH domains. The crystal structures of Lpd cc-RA$\mathrm{PH}$ and Lpd RA-PH were both solved to $2.4 \AA$, with cc-RA-PH in $\mathrm{C} 2$ space group and $\mathrm{RA}-\mathrm{PH}$ in $\mathrm{P} 2{ }_{1}$ space group. Both structures were determined by molecular replacement using RIAM RA-PH domains (PDB 3TCA) as the searching model (Wynne et al., in press). The asymmetric unit of Lpd cc-RA-PH contains 
Table 1. Data collection and refinement statistics for Lpd RA-PH and Lpd cc-RA-PH

\begin{tabular}{|c|c|c|}
\hline & Lpd RA-PH & Lpd cc-RA-PH \\
\hline \multicolumn{3}{|l|}{ Data collection } \\
\hline Space group & $P 2_{1}$ & $\mathrm{C} 2$ \\
\hline$a, b, c(\AA)$ & $75.3,93.5,80.7$ & $117.6,97.0,76.8$ \\
\hline$\alpha, \beta, \gamma\left(^{\circ}\right)$ & $90.0,104.9,90.0$ & $90.0,127.0,90.0$ \\
\hline Resolution ( $\AA$ ) & $50.0-2.4$ & $50.0-2.4$ \\
\hline Completeness (\%) & $98.6(99.2)$ & 95.7 (79.9) \\
\hline$R_{\text {sym }}(\%)$ & $0.064(0.261)$ & $0.071(0.380)$ \\
\hline$I / \sigma(I)$ & $23(5.4)$ & $17.6(2.4)$ \\
\hline Unique reflections & 41656 & 25743 \\
\hline Redundancy & $2.9(2.9)$ & $3.7(3.4)$ \\
\hline \multicolumn{3}{|l|}{ Refinement } \\
\hline$R_{\text {work }}(\%)$ & 25.3 & 23.8 \\
\hline$R_{\text {free }}(\%)$ & 30.3 & 28.1 \\
\hline RMSD bonds $(\AA)$ & 0.010 & 0.007 \\
\hline RMSD angle $\left({ }^{\circ}\right)$ & 1.409 & 1.148 \\
\hline Protein atoms & 8204 & 4357 \\
\hline Solvent atoms & 249 & 103 \\
\hline Total residues & 1020 & 562 \\
\hline \multicolumn{3}{|l|}{ Average $B$-factors $\left(\AA^{2}\right)$} \\
\hline Main chain atoms & 18.1 & 22.8 \\
\hline Side chain atoms & 18.7 & 22.8 \\
\hline Solvent & 27.8 & 35.0 \\
\hline \multicolumn{3}{|l|}{ Ramachandran } \\
\hline Favored regions (\%) & 96.2 & 98.1 \\
\hline Allowed regions (\%) & 100.0 & 100.0 \\
\hline
\end{tabular}

two molecules, while RA-PH contains four molecules. A superposition of cc-RA-PH and RA-PH shows a virtually identical overall architecture with an rmsd of $0.78 \AA$ over $243 C_{\alpha}$ atoms. Both RA and $\mathrm{PH}$ domains share the conventional folds of their families. As seen in Grb10 and RIAM, RA and PH domains unite to form an integrated structural module (Depetris et al., 2009; Wynne et al., 2012). Particularly, the coiled-coil regions of the two cc-RA-PH molecules in the asymmetric unit, related by a pseudo two-fold symmetry, form an anti-parallel coiled-coil interaction (Fig. 1B). Data collection and refinement statistics are listed in Table 1.

\section{Lpd oligomerizes through a coiled-coil interaction}

Structural analysis of cc-RA-PH revealed a coiled-coil interaction between the two molecules at the $\mathrm{N}$-terminus (Fig. 1B). The two helices are positioned as an anti-parallel pair with several hydrophobic residues including Ile256, Ala259, Ile263 and Ala266 positioned alternatively at each helical turn, defining a hydrophobic contact interface. These residues align in a zigzag fashion and form Ala:lle interacting pairs (Fig. 1C). In addition, the side-chain of isoleucines also interacts with the pairing isoleucines of the other molecule to further fortify this coiledcoil hydrophobic contact. In the crystal structure of a RIAM construct (hereafter referred to as RIAMs to avoid possible confusion with Lpd RA-PH) equivalent to Lpd cc-RA-PH, the putative coiled-coil region is completely disordered, possibly due to an unfavorable replacement of the lle263 (in Lpd) by a leucine residue in RIAM towards the carboxyl end of the helix (Wynne et al., in press). Interestingly, this Lpd dimer is in close contact with another symmetry-related dimer through coiledcoil stacking. The two helical pairs formed a cross pattern with a $67^{\circ}$ rotation (Fig. S1). The two helices of molecules A and B are 8.4 $\AA$ apart (measured from the helical axis), and this helical pair is $11.7 \AA$ from the symmetry-related helical pair of molecules A" and B" (Fig. S1). The two helical pairs make contact through three hydrophobic interactions and a hydrogen bond: Ile256(A) to Leu260(B"), Leu260(B) to Ile256(A"), Ile263(B) to 
Ile263(B"), and Gln267(B) to Gln267(B"), respectively.

\section{Structural comparison reveals a second intermolecular contact site}

In addition to the coiled-coil region, in both cc-RA-PH and RA$\mathrm{PH}$ structures, a common intermolecular contact site was observed, strongly indicating a physiological relevance. This interface is located between the N-terminus of the RA domain B1 strand in molecule A and the RA-PH linker in molecule B', which is symmetrically related to molecule $B$ (Fig. $2 A$ ). Molecules $A$ and $B$ ' make contact through a hydrophobic interaction formed by Val272 of molecule A and Leu368,Ala374, and Met376 of molecule B'. This interaction is further reinforced by backbone hydrogen bonds, in which the backbone amide of Val272 and carboxyl group of Lys270 of molecule A interact with the carboxyl group and backbone amide of Glu375 of molecule B', respectively (Fig. 2B). The local conformation of this contact site is quite stable as shown by the well-defined electronic density map (Fig. S2A). Further solution analysis showed that this interface is not due to crystal packing, nor the coiled-coil interface. Residues involved in this contact site are not conserved in RIAM or in other MRL proteins, suggesting a unique oligomerization configuration of Lpd (Fig. S3). This RA:linker interface and the coiled-coil interface implicate a molecular configuration for Lpd oligomerization that is essential for actin-binding protein clustering and subsequent actin rearrangement.

The RA domain of Lpd adopts a typical ubiquitin-like structural fold, similar to the RA domain or RBD domain of other Ras binding proteins (Fig. S2B). As shown in the crystal structure of other GTPase:RA/RBD domain complexes such as cRaf (PDB 1GUA), RalGDS (PDB 1LFD), PI3KY (PDB 1HE8), and $P L C \varepsilon$ (PDB 2C5L), the primary interaction site is between the $\beta 2$ strand of RA/RBD domain and the switch I region of active Ras GTPase through $\beta$ sheet packing and salt bridging (Fig. 2C, S2B) (Nassar et al., 1996; Huang et al., 1998; Pacold et al., 2000; Bunney et al., 2006). However, when we superimposed the Lpd cc-RA-PH non-crystallographic dimer (molecule $A$ and $B^{\prime}$ ) formed via the RA:linker interface with the structure of PLCE:Ras complex, the putative Ras binding site on molecule $A$ is partially occupied by molecule $B$ ' in the dimer (Fig. 2C), suggesting that oligomerization of Lpd through the RA:linker interface may interfere with its binding to Ras family GTPase.

\section{Validation of Lpd intermolecular contacts in solution by mutagenesis study}

Dimerization or higher-order oligomerization of protein molecules often plays an important role in enhancing their biological functions through an increased binding avidity (Stein et al., 2003; Shiroishi et al., 2006; Depetris et al., 2009; Arai et al., 2012; McElroy et al., 2012). To validate the biological relevancy of the two contact sites observed from the crystal structures, mutagenesis and gel filtration chromatography were conducted to monitor oligomerization states in solution. First, we compared Lpd cc-RA-PH with Lpd RA-PH, RIAMs, and BSA (Fig. 3A). The elution profile of Lpd cc-RA-PH (MW $33 \mathrm{kDa}$ ) shows a sharp monomer peak at $13.2 \mathrm{~mL}$ and a broad peak that overlaps with both the BSA monomer $(67 \mathrm{kDa})$ and dimer (134 kDa) peaks (Fig. 3A, embedded panel). This result suggests Lpd not only can dimerize, but can also form higherorder oligomers in solution. The equivalent RIAMs protein does not form dimer under the same loading concentration. Interestingly, the RA-PH domains of Grb10 also form dimer under a much higher loading concentration $(>20 \mathrm{mg} / \mathrm{mL}$ ) (Depetris et al., 2009). Evidently, the $K_{d}$ for Lpd dimerization in solution is much lower than that of Grb10, due to the combined effort of both the coiled-coil interaction and the RA:linker interaction. In the absence of the coiled-coil region, dimerization is significantly suppressed as seen in the Lpd RA-PH elution profile (Fig. 3A). It appears that without the coiled-coil interaction, the RA:linker interaction relatively weak and undetectable under a moderate concentration.

We then mutated Ala259 in the coiled-coil interface and measured oligomerization in solution. The A259S mutation significantly reduced the oligomerization of $\mathrm{Lpd}$, and a more aggressive mutation A259Y completely diminished it (Fig. 3B). Similarly, two RA:linker interface-disrupting mutations V272Y and $\mathrm{A} 374 \mathrm{Y}$ also abrogated the oligomerization of $\mathrm{Lpd}$, despite the coiled-coil region remaining intact, suggesting that this interaction may also help stabilize the $\mathrm{N}$-terminal helical region for the coiled-coil interaction. The diminished oligomerization of Lpd was verified by SDS-PAGE (Fig. 3B, embedded panel). Overall, the loss of oligomerization in these single mutations suggests that the two interfaces are not independent, but function coordinately by mutually stabilizing anda favorable local conformation for the interactions.

\section{Phosphoinositide binding to Lpd}

The PH domain often serves a common membrane translocalization function by binding to membrane phosphoinositide. In RA-PH containing proteins, $\mathrm{PH}$ anchoring to the membrane also facilitates the interaction with the membrane-locating Ras GTPase, thereby enhancing the relevant signaling events (Depetris et al., 2009; Wynne et al., in press). We measured the phosphoinositide-binding affinities of Lpd cc-RA-PH using various fluorescently labeled phosphoinositides in a florescencepolarization assay (Ceccarelli et al., 2007). Only $\mathrm{PI}(3,4,5) \mathrm{P}_{3}$, $\mathrm{PI}(4,5) \mathrm{P}_{2}, \mathrm{PI}(3,4) \mathrm{P}_{2}$, and $\mathrm{PI}(5) \mathrm{P}$ bind $\mathrm{Lpd}$ with low affinity $\left(K_{\mathrm{d}} \geq 29 \mu \mathrm{mol} / \mathrm{L}\right)$ (Fig. 4C).

In a canonical binding mode to phosphoinositide, the $\mathrm{PH}$ domain interacts with the headgroup through the $\beta 1-\beta 2$ loop, which harbors a consensus sequence of K-Xn-(K/R)-X-R. Sequence and structure alignment of $L p d$ and a canonically binding PH domain of Grp1 reveal that Lys410, Lys414 and Arg416 of Lpd reassemble this motif, and adopt similar configurations as Grp1 (Fig. 4A and 4B). The Grb10 PH domain binds to headgroups in a noncanonical mode through interactions with 
A

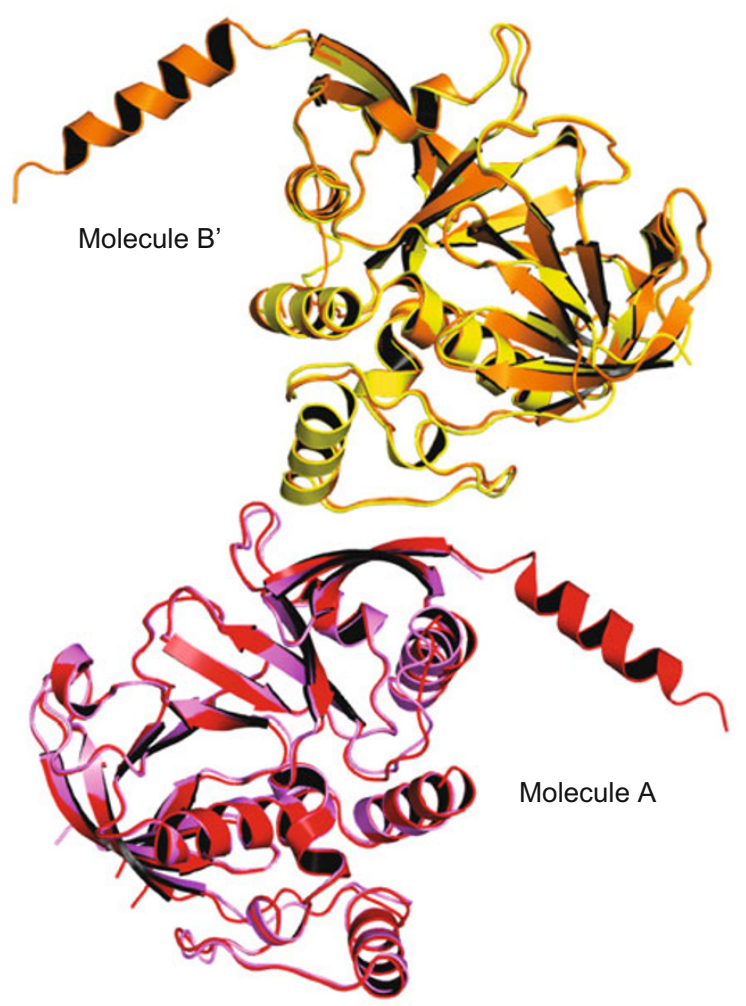

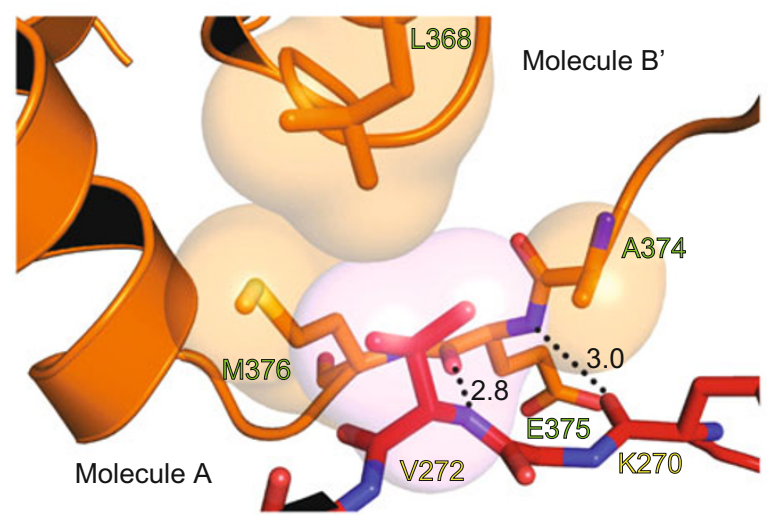

C

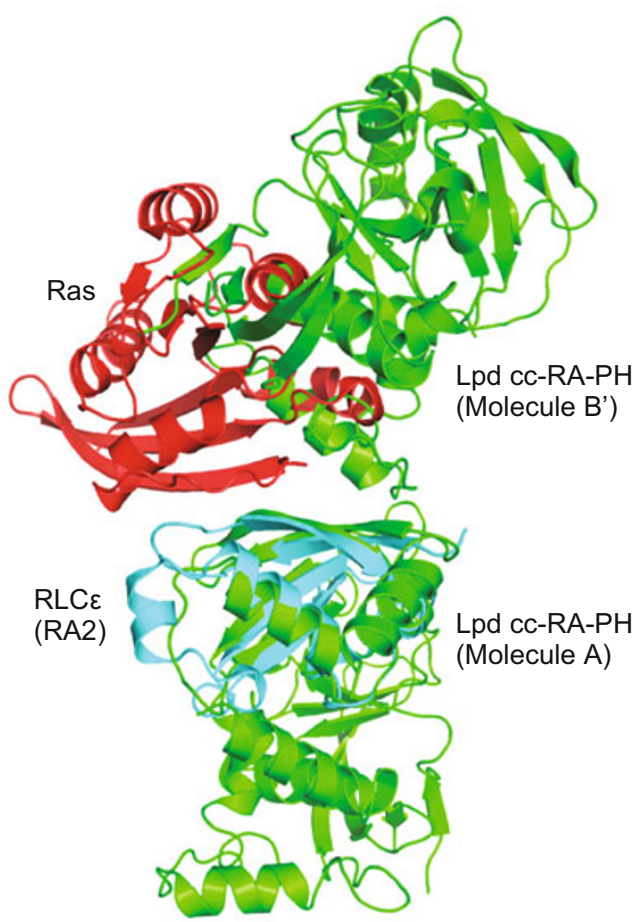

Figure 2. A second intermolecular contact site was identified via an RA:linker interaction. (A) Superposition of RA-PH and cC-RA$\mathrm{PH}$ structures revealed a common intermolecular interface via an RA:linker contact site. The two cc-RA-PH molecules (A and B') forming a non-crystallographic dimer are in orange and red, and the two RA-PH molecules are in yellow and pink. (B) Stick model of the secondary contact site between molecules A and B' of Lpd cc-RA-PH illustrates the hydrophobic interactions between Val272 of molecule A with residues Leu368, Ala374, and Met376 of molecule B', and backbone H-bond interactions. Hydrogen bonds are denoted by dotted line with their distances in angstrom. We used the same color scheme for the two molecules as in Fig. 2A. (C) Superposition of a cc-RA-PH dimer via the RA:linker interface and a PLC $\varepsilon$ :Ras complex (PDB 2C5L) reveals a steric conflict between Lpd molecule B' and Ras. Green: cc-RA-PH; cyan: PLCE RA2; red: Ras.

the $\beta 5-\beta 6$ loop, likely due to the absence of the crucial Arg416 residue in the $\beta 1-\beta 2$ loop (Leu252 in Grb10). Furthermore, mutations of Lys331 or Arg333 in the RIAM $\beta 1-\beta 2$ loop (Lys414 or Arg416 in Lpd) diminished the plasma membrane translocation of RIAM (Wynne et al., in press). It is possible that Lpd's (and RIAM's) lower affinity to headgroups is attributed to the presence of two negatively charged residues (Asp407 and Asp408) in the $\beta 1-\beta 2$ loop. To test this, we mutated the two aspartate residues to glycines based on the Grp1 sequence in an attempt to increase the canonical binding. Indeed, we observed a moderate 1.7-fold increase in $\mathrm{PI}(3,4,5) \mathrm{P} 3$ binding (Fig. 4D). In the case of Grb10, a non-canonical binder, mutations of the corresponding residues (Glu243 and Leu244, Fig. 4A) did not show any change in headgroup binding (Depetris et al., 2009).

\section{DISCUSSION}

The crystal structure of Lpd cc-RA-PH presents a molecular model of how Lpd may enhance actin polymerization by oligomerization via two intermolecular contact sites. Further- 
A

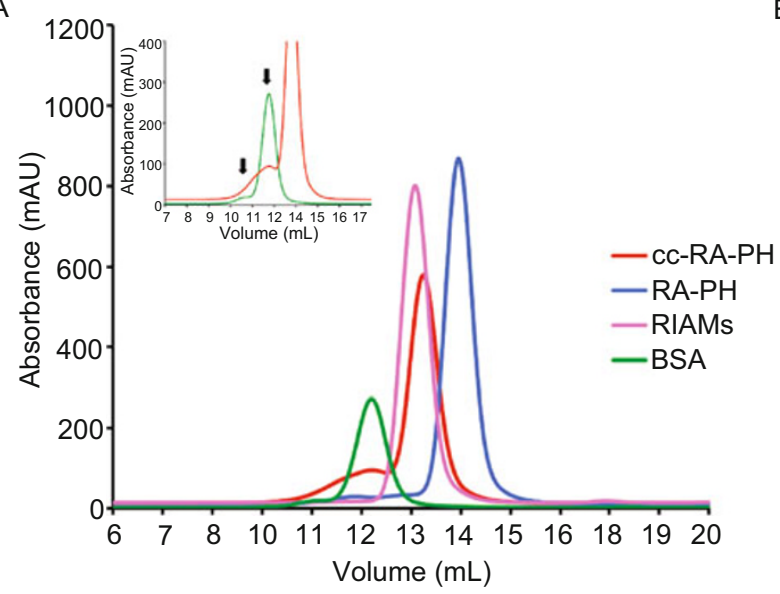

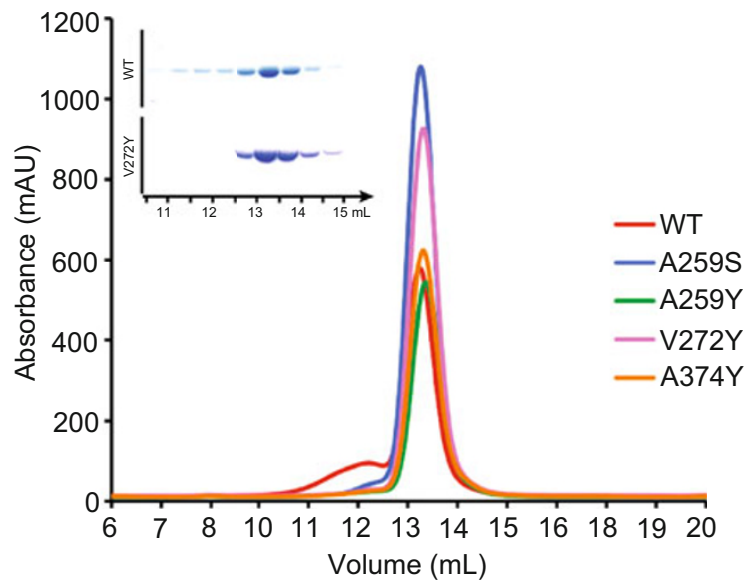

Figure 3. Oligomerization analysis of Lpd in solution by gel filtration chromatography. (A) Comparison of elution profiles of Lpd RA-PH, Lpd cc-RA-PH, RIAMs (Lpd cc-RA-PH equivalent), and BSA. Embedded panel shows the oligomer peak of Lpd cc-RA-PH in comparison with BSA trace. The two arrows mark the elution volumes for Lpd dimer (BSA monomer) and Lpd tetramer (BSA dimer). (B) Compilation of elution profile of Lpd cc-RA-PH wild-type and point mutations at the two intermolecular contact sites. All mutations exhibit the loss (or reduction) of the oligomer peak. Protein component of the fractions from Lpd cc-RA-PH and V272Y is shown in the embedded SDS-PAGE.

more, it is possible that the coiled-coil stacking interaction may extend the Lpd oligomerization (Fig. S1). Interestingly, the homologous RIAM molecule lacks these interactions in both crystallographic and solution studies. Ile263 in Lpd forms the hydrophobic interface with Ile256 and Ala259 at the coiledcoil contact site. The coiled-coil contact in RIAM may be suppressed due to the substitution of lle263 by a leucine residue at the corresponding position, as leucine's longer side-chain branch may extend the hydrophobic contact distance between the two coiled-coil helices. The RA:linker contact site is also missing in RIAM, resulting in total absence of oligomerization. Although RIAM is predicted to have a second coiled-coil region, a RIAM construct containing both coiled-coil regions with the RA-PH unit did not show any detectable oligomerization in solution (data not shown). Both Lpd and RIAM are ligands of Ena/VASP proteins, and overexpression of either Lpd or RIAM induces lamellipodia formation. However, only Lpd knockdown cells show impaired lamellipodia formation, whereas RIAM knockdown only affects F-actin content (Krause et al., 2004). Our present study supports these results and demonstrates a molecular mechanism by which Lpd may play an overwhelmingly essential role in lamellipodia formation and actin polymerization.

Both MRL and Grb7/10/14 family proteins contain a signature RA-PH tandem domain configuration. This RA-PH configuration is required to maintain the integrity of both domains and also to coordinate their distinct functions of Ras signaling and membrane translocalization. Binding affinity of the RA domain in the integrated RA-PH unit to Ras GTPase is weaker compared to that of other independent Ras binding domains (Nassar et al., 1996; Huang et al., 1998; Rodriguez-Viciana et al., 2004). The low affinity to the membrane-anchoring Ras GT-
Pase can be compensated by the membrane translocalization via the PH domain (Holt and Daly, 2005; Depetris et al., 2009; Wynne et al., in press). Grb10 interacts with Ras and regulates insulin signaling, whereas RIAM can be recruited by Rap1 and mediates integrin activation. Both insulin receptor and integrin are transmembrane proteins. Thus, membrane localization capability is crucial for these adapter-signaling molecules (namely, Grb10 and RIAM) that mediate these pathways. Compared to other independent $\mathrm{PH}$ domains, binding of RA-PH to phosphoinositide is moderate. In the case of Lpd, the phosphoinositide binding is further weakened. Despite high sequence identity, the $K_{d}$ of $L p d$ for phosphoinositide binding is much higher than that of Grb10 (6-fold) and RIAM (2-fold), close to the estimated membrane phosphoinositide concentration (Winks et al., 2005). It is possible that the glutamate residue in RIAM corresponding to Asp407 in Lpd may be more flexible and adopts a conformation that avoids direct conflict with the phosphate group of the bound phosphoinositide (Fig. 4A). In addition, the KRR sequence of RIAM in $\beta 2$ may be more accessible than the KKR sequence in Lpd for a negatively charged phosphoinositide. The lack of phosphoinositide binding of Lpd may also affect its Ras signaling. Although the GTPase binding site of Lpd and RIAM is virtually identical, neither Rap1 nor other Ras GTPases has been identified as the signaling partner of Lpd, possibly due to the insufficient compensation from $\mathrm{PH}$-domainmediated membrane translocalization. In fact, Lpd retains the capability of binding to talin, the cytoskeletal protein that directly activates integrins (Calderwood et al., 1999; Wegener et al., 2007). But its degenerated Rap1 binding affinity and membrane translocalization may have diminished its role in integrinmediated cell adhesion.

Mammalian cells express both Lpd and RIAM proteins. Do 
A
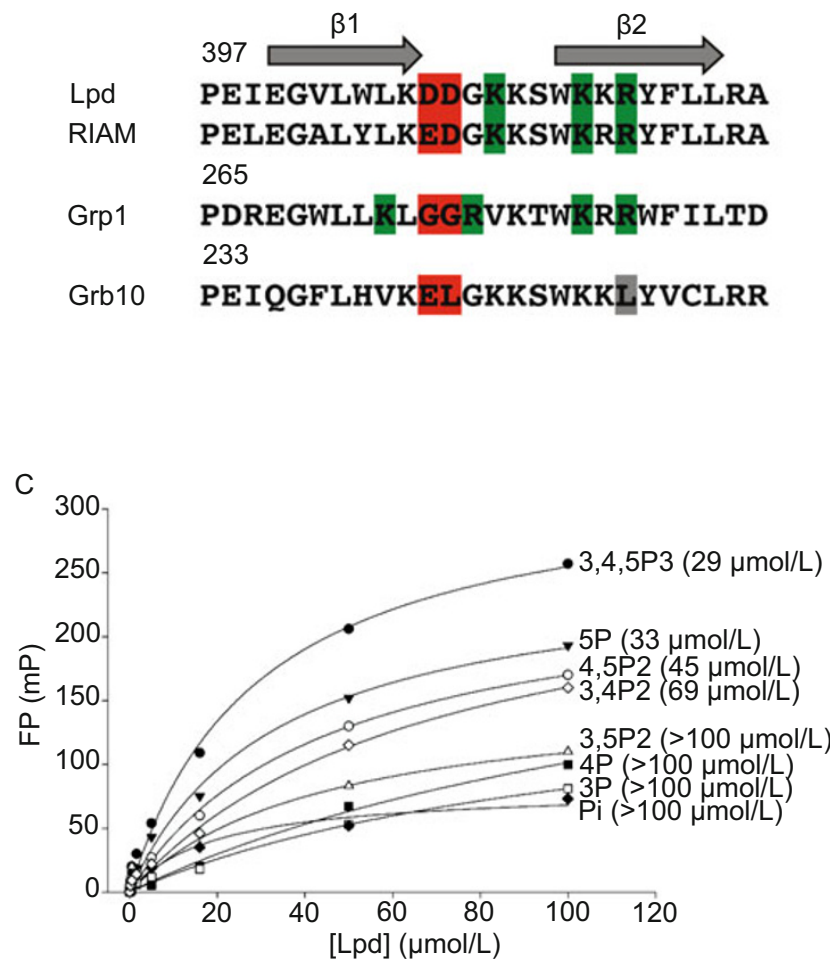

B
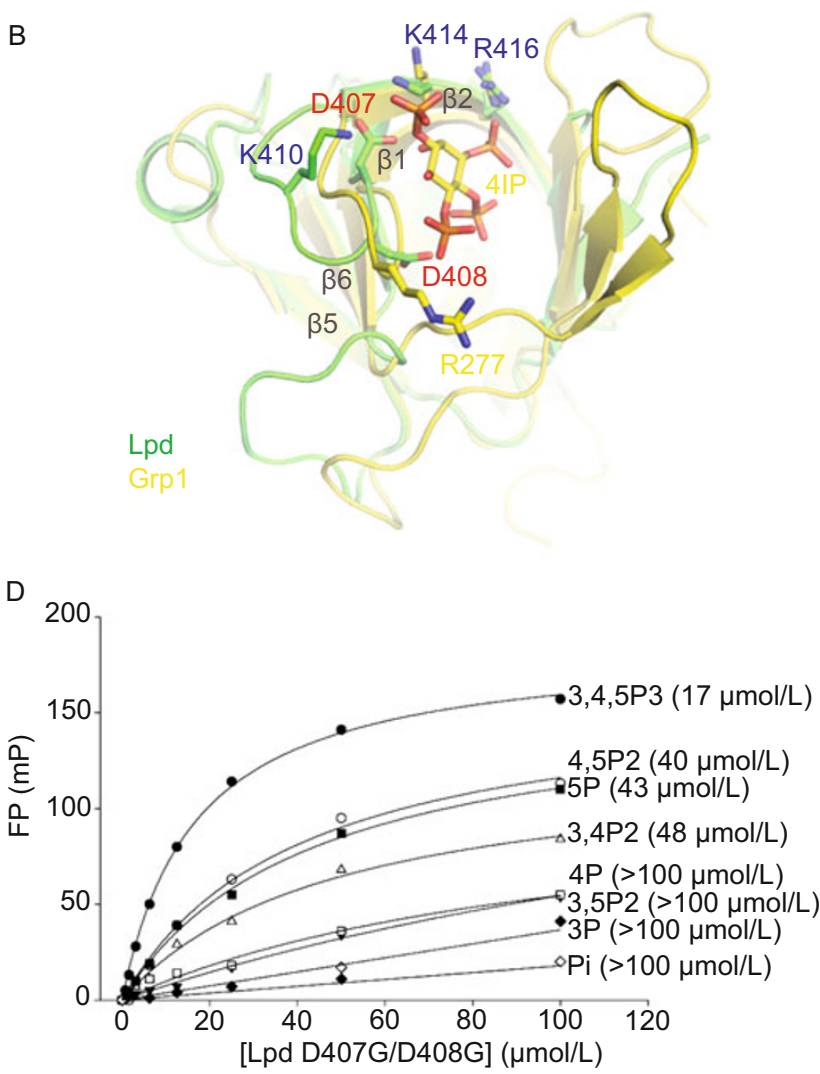

Figure 4. Phosphoinositide binding to Lpd. (A) Sequence alignment of the RA (RBD) $\beta 1-\beta 2$ loop of Lpd, RIAM, Grp1, and Grb10. PIPinteracting residues are highlighted in green. Mutations made to improve headgroup binding in Lpd (Asp407 and Asp408) are highlighted in red. Leu252 of Grb10, highlighted in grey, prevents the canonical binding mode of phosphoinositide. (B) Superposition of Grp1:4IP complex (yellow) and the PH domain of Lpd (green). Residues predicted to interact with headgroup are labeled in blue. Asp407 and Asp408 are labeled in red. (C) Fluorescence-polarization measurements of phosphoinositide binding to wild-type Lpd. Fits are based on a saturable, single-site binding model. $K_{d}$ values extracted from the fits are given in parentheses to the right of the headgroup labels. (D) Same as (C) but for Lpd D407G/D408G mutant.

these two homologous proteins serve redundant functions? Or have their functions diverged during evolution? We demonstrate here that Lpd is capable of forming high-order oligomers and its affinity to phosphoinositide is much weaker than that of RIAM. Along with previous structural and biochemical data and in-cell characterization, our current data lead to a working model that Lpd and RIAM serve diverse functions: Lpd predominately functions in enhancing actin polymerization and regulating cell migration, whereas the primary function of RIAM is to mediate Rap1-induced cell adhesion.

\section{MATERIALS AND METHODS}

Plasmid construction and protein purification

The Lpd constructs were cloned into pET28a expression vector with a His6 and a TEV protease cleavage site. The two constructs, RA-PH and cc-RA-PH, consist of amino acid residues from 266 to 520 and 240 to 520 , respectively. Lpd point mutants were constructed using site-directed mutagenesis method. Plasmids were transformed into Escherichia coli BL21(DE3) for protein expression. Cells were grown in $37^{\circ} \mathrm{C}$ LB medium containing $50 \mu \mathrm{g} / \mathrm{mL}$ kanamycin until $A 600$ reached
$0.6-0.7$, and induced by addition of $0.1 \mathrm{mmol} / \mathrm{L}$ IPTG at $16^{\circ} \mathrm{C}$. Protein purifications were carried out at $4^{\circ} \mathrm{C}$. The cell pellet was resuspended in $20 \mathrm{mmol} / \mathrm{L}$ Tris, $\mathrm{pH} 7.5$ and $500 \mathrm{mmol} / \mathrm{L} \mathrm{NaCl}$ then lysed with a homogenizer EmulsiFlex-C3 (AVESTIN, Inc.). Protein sample was extracted from the supernatant using a HisTrap FF column (GE Healthcare). Histidine tag was removed by incubating with TEV protease. Lpd constructs, wild-type and mutants, were subsequently purified by ion exchange chromatography using a Resource $S$ column (GE Healthcare).

Solution characterization by gel filtration chromatography

Purified proteins of interest were concentrated to $2-3 \mathrm{mg} / \mathrm{mL}$ except Lpd cc-RA-PH $(1.8 \mathrm{mg} / \mathrm{mL})$ and BSA $(1 \mathrm{mg} / \mathrm{mL})$, and subject to gel filtration analysis. The experiment was performed using a Superose 12 10/300 GL column (GE Healthcare) with $20 \mathrm{mmol} / \mathrm{L}$ HEPES, pH 7.0 and $500 \mathrm{mmol} / \mathrm{L} \mathrm{NaCl}$. Fractions were collected and resolved on the SDS-PAGE.

X-ray crystallography

Lpd RA-PH and cc-RA-PH were crystallized by hanging-drop vapor dif- 
fusion method at room temperature. Each crystallization drop contained equal volumes of protein and reservoir solution. RA-PH $(7.6 \mathrm{mg} / \mathrm{mL})$ was crystallized in $200 \mathrm{mmol} / \mathrm{L}$ sodium malonate, $\mathrm{pH} 5.0$ and $20 \%(\mathrm{w} / \mathrm{v})$ polyethylene glycol 3350 . cc-RA-PH $(5.5 \mathrm{mg} / \mathrm{mL})$ was crystallized in 50 $\mathrm{mmol} / \mathrm{L}$ sodium formate, $20 \%(\mathrm{w} / \mathrm{V})$ polyethylene glycol 3350 , and $30 \%$ $(v / v)$ ethylene glycol. The crystals were allowed to grow for 1-2 days prior to diffraction experiments. Dehydration step was performed with addition of $5 \%(w / v)$ polyethylene glycol 3350 to the reservoir solution. RA-PH crystals were briefly soaked in reservoir solution containing $20 \%(V / V)$ ethylene glycol as cryoprotectant, then flash-frozen in a 100$\mathrm{K}$ stream of gaseous nitrogen.

X-ray diffraction data for Lpd RA-PH and cc-RA-PH crystals were collected at the beamlines $\mathrm{X} 25$ and $\mathrm{X} 4 \mathrm{C}$ of the National Synchrotron Light Source at Brookhaven National Laboratory (Upton, NY), respectively. Data were processed using the HKL-2000 package (Otwinowski and Minor, 1997). The initial phases of Lpd RA-PH and cc-RA-PH were determined using molecular replacement method with the RA-PH domain of RIAM (PDB 3TCA) as the initial model. Structural refinement was performed using REFMAC (Murshudov et al., 1997) and Coot (Emsley and Cowtan, 2004). The models were validated with SFCHECK (Vaguine et al., 1999) and MolProbity (http://molprobity.biochem.duke. edu/). Data collection and refinement statistics are listed in Table 1. Structural figures were generated using PyMOL program suite (http:// pymol.sourceforge.net).

\section{Phosphoinositide binding measured by fluorescent polarization}

Purified Lpd cc-RA-PH or its mutant D407G/D408G was mixed with $12.5 \mathrm{nmol} / \mathrm{L}$ of BODIPY TMR-labeled phosphoinositides (Echelon Biosciences Incorporated) in a buffer containing $20 \mathrm{mmol} / \mathrm{L}$ HEPES $\mathrm{pH} 7.5,150 \mathrm{mmol} / \mathrm{L} \mathrm{NaCl}$ and $0.05 \%(\mathrm{~V} / \mathrm{V})$ Tween-20 at concentrations ranging from $0.8 \mu \mathrm{mol} / \mathrm{L}$ to $100 \mu \mathrm{mol} / \mathrm{L}$. After $5 \mathrm{~min}$ of incubation, the reaction mixes were measured for fluorescence polarization at room temperature on a TECAN Infinite F500 plate reader (Tecan Group Ltd.). A 535/25 nm filter was used as an excitation filter, and a pair of $590 / 20 \mathrm{~nm}$ filters were used as emission polarization filters. Data were processed and fitted to a single-site (saturating) binding model using SigmaPlot (Systat Software).

\section{ACCESSION CODES}

Protein Data Bank: Atomic coordinates and structure factors for Lpd RA-PH and Lpd cc-RA-PH have been deposited with the accession codes 4GN1 and 4GMV.

\section{ACKNOWLEDGMENTS}

This work was supported by Fox Chase Cancer Center startup fund (to J.W). We thank Dr. Stevan Hubbard (NYU School of Medicine) for providing lamellipodin cDNA and instrumental support, and for insightful discussion, supporting staff at beamlines X4C and X25 at the National Synchrotron Light Source, Brookhaven National Laboratory.

Y.C.C and J.W performed crystallographic studies, structure refinement, biochemical experiments and manuscript preparation; J.W. performed fluorescence-polarization assays; H.Z. performed crystallographic studies and mutagenesis studies; M.L.B. provided technical support and performed manuscript preparation. J.W. supervised the project and was the principal manuscript author.

\section{REFERENCES}

Arai, S., Yonezawa, Y., Okazaki, N., Matsumoto, F., Tamada, T., Tokunaga, H., Ishibashi, M., Blaber, M., Tokunaga, M., and Kuroki, R. (2012). A structural mechanism for dimeric to tetrameric oligomer conversion in Halomonas sp. nucleoside diphosphate kinase. Protein Sci 21, 498-510.

Bunney, T.D., Harris, R., Gandarillas, N.L., Josephs, M.B., Roe, S.M., Sorli, S.C., Paterson, H.F., Rodrigues-Lima, F., Esposito, D., Ponting, C.P., et al. (2006). Structural and mechanistic insights into ras association domains of phospholipase $C$ epsilon. Mol Cell 21, 495-507.

Calderwood, D.A., Zent, R., Grant, R., Rees, D.J., Hynes, R.O., and Ginsberg, M.H. (1999). The Talin head domain binds to integrin beta subunit cytoplasmic tails and regulates integrin activation. J Biol Chem 274, 28071-28074.

Ceccarelli, D.F., Blasutig, I.M., Goudreault, M., Li, Z., Ruston, J., Pawson, T., and Sicheri, F. (2007). Non-canonical interaction of phosphoinositides with pleckstrin homology domains of Tiam1 and ArhGAP9. J Biol Chem 282, 13864-13874.

Colo, G.P., Lafuente, E.M., and Teixido, J. (2012). The MRL proteins: Adapting cell adhesion, migration and growth. Eur J Cell Biol. (In Press).

Depetris, R.S., Wu, J., and Hubbard, S.R. (2009). Structural and functional studies of the Ras-associating and pleckstrin-homology domains of Grb10 and Grb14. Nat Struct Mol Biol 16, 833-839.

Emsley, P., and Cowtan, K. (2004). Coot: model-building tools for molecular graphics. Acta Crystallogr D Biol Crystallogr 60, 2126-2132.

Holt, L.J., and Daly, R.J. (2005). Adapter protein connections: the MRL and Grb7 protein families. Growth Factors 23, 193-201.

Huang, L., Hofer, F., Martin, G.S., and Kim, S.H. (1998). Structural basis for the interaction of Ras with RalGDS. Nat Struct Biol 5, 422-426.

Krause, M., Dent, E.W., Bear, J.E., Loureiro, J.J., and Gertler, F.B. (2003). Ena/VASP proteins: regulators of the actin cytoskeleton and cell migration. Annu Rev Cell Dev Biol 19, 541-564.

Krause, M., Leslie, J.D., Stewart, M., Lafuente, E.M., Valderrama, F., Jagannathan, R., Strasser, G.A., Rubinson, D.A., Liu, H., Way, M., et al. (2004). Lamellipodin, an Ena/NASP ligand, is implicated in the regulation of lamellipodial dynamics. Dev Cell 7, 571-583.

Lafuente, E.M., van Puijenbroek, A.A., Krause, M., Carman, C.V., Freeman, G.J., Berezovskaya, A., Constantine, E., Springer, T.A., Gertler, F.B., and Boussiotis, V.A. (2004). RIAM, an Ena/VASP and Profilin ligand, interacts with Rap1-GTP and mediates Rap1induced adhesion. Dev Cell 7, 585-595.

Lee, H.S., Lim, C.J., Puzon-McLaughlin, W., Shattil, S.J., and Ginsberg, M.H. (2009). RIAM activates integrins by linking talin to ras GTPase membrane-targeting sequences. J Biol Chem 284, 5119-5127.

Li, H., Schopfer, L.M., Masson, P., and Lockridge, O. (2008). Lamellipodin proline rich peptides associated with native plasma butyrylcholinesterase tetramers. Biochem J 411, 425-432.

McElroy, C.A., Holland, P.J., Zhao, P., Lim, J.M., Wells, L., Eisenstein, E., and Walsh, S.T. (2012). Structural reorganization of the interleukin-7 signaling complex. Proc Natl Acad Sci U S A 109, 2503-2508.

Murshudov, G.N., Vagin, A.A., and Dodson, E.J. (1997). Refinement of macromolecular structures by the maximum-likelihood method. Acta Crystallogr D Biol Crystallogr 53, 240-255. 
Nassar, N., Horn, G., Herrmann, C., Block, C., Janknecht, R., and Wittinghofer, A. (1996). Ras/Rap effector specificity determined by charge reversal. Nat Struct Biol 3, 723-729.

Otwinowski, Z., and Minor, W. (1997). Processing of X-ray diffraction data collected in oscillation mode. Methods Enzymol 276, 307-326.

Pacold, M.E., Suire, S., Perisic, O., Lara-Gonzalez, S., Davis, C.T., Walker, E.H., Hawkins, P.T., Stephens, L., Eccleston, J.F., and Williams, R.L. (2000). Crystal structure and functional analysis of Ras binding to its effector phosphoinositide 3-kinase gamma. Cell 103, 931-943.

Rodriguez-Viciana, P., Sabatier, C., and McCormick, F. (2004). Signaling specificity by Ras family GTPases is determined by the full spectrum of effectors they regulate. Mol Cell Biol 24, 4943-4954.

Shiroishi, M., Kuroki, K., Ose, T., Rasubala, L., Shiratori, I., Arase, H., Tsumoto, K., Kumagai, I., Kohda, D., and Maenaka, K. (2006). Efficient leukocyte Ig-like receptor signaling and crystal structure of disulfide-linked HLA-G dimer. J Biol Chem 281, 10439-10447.

Stein, E.G., Ghirlando, R., and Hubbard, S.R. (2003). Structural basis for dimerization of the Grb10 Src homology 2 domain. Implications for ligand specificity. J Biol Chem 278, 13257-13264.

Vaguine, A.A., Richelle, J., and Wodak, S.J. (1999). SFCHECK: a unified set of procedures for evaluating the quality of macromolecular structure-factor data and their agreement with the atomic model. Acta Crystallogr D Biol Crystallogr 55, 191-205.

Wegener, K.L., Partridge, A.W., Han, J., Pickford, A.R., Liddington, R.C., Ginsberg, M.H., and Campbell, I.D. (2007). Structural basis of integrin activation by talin. Cell 128, 171-182.

Winks, J.S., Hughes, S., Filippov, A.K., Tatulian, L., Abogadie, F.C., Brown, D.A., and Marsh, S.J. (2005). Relationship between membrane phosphatidylinositol-4,5-bisphosphate and receptormediated inhibition of native neuronal M channels. J Neurosci 25, 3400-3413.

Wynne, J.P., Wu, J., Su, W., Mor, A., Patsoukis, N., Boussiotis, V.A., Hubbard, S.R., and Philips, M.R. (2012). Rap1-Interacting Adapter Molecule (RIAM) Associates with the Plasma Membrane via a Proximity Detector. J Cell Biol. (In Press). 